DOI 10.17816/transsyst201843s1272-278

\author{
(C) L. Fang, Y. He, L. Zhang \\ CRRC Zhuzhou Electric Motor Co., Ltd \\ (Zhuzhou, Hunan, China)
}

\title{
RESEARCH ON MECHANISM OF EDDY CURRENT IN RAIL OF MAGLEV AND OPTIMUM DESIGN OF ELECTROMAGNET
}

\begin{abstract}
Aim: Due to the movement of medium-low speed maglev vehicles, eddy current is generated in the rail, leading to reduced levitation force. This detrimental effect becomes more prominent with increasing velocity. In order to reduce the influence of eddy current effect, the electromagnet is optimized to meet the requirement of vehicle speeding up from $100 \mathrm{~km} / \mathrm{h}$ to $160 \mathrm{~km} / \mathrm{h}$.

Methods of the studies: To maintain a constant levitation force, the current must also increase accordingly resulting in higher power consumption and heat generation. In this paper, a mathematical model is established by analytical method, focusing on the mechanism and influencing factors of eddy current. Three-dimensional transient magnetic field magnet model is analyzed by the ANSYS electromagnetic simulation software Maxwell.

Results: The levitation force is related to five parameters, such as speed, length of the electromagnet, rail height, rail thickness and air gap. According to the finite element simulation results, when the train speed is $160 \mathrm{~km} / \mathrm{h}$, the levitation force of the end electromagnet is reduced by about $21.9 \%$. The levitation force of optimized electromagnet increases by $27 \%$ under the same current, which can compensate for the drop of levitation force caused by eddy current. The levitation force is $41.4 \mathrm{kN}$ at the speed of $160 \mathrm{~km} / \mathrm{h}$, which is slightly larger than the $39.6 \mathrm{kN}$ of the former electromagnet static levitation force, which can meet the requirements.

Conclusion: The result confirms that optimization methods proposed above are valid and effective.
\end{abstract} force

Keywords: Medium-low speed maglev vehicles; Electromagnet; Eddy current; Levitation

\section{INTRODUCTION}

Electromagnetic levitation and linear induction motor systems are commonly used in medium and low-speed maglev systems. China's first medium and low speed commercial line "Changsha maglev express line" was officially opened and operated at a speed of $100 \mathrm{~km} / \mathrm{h}$ in May 2016. Another $160 \mathrm{~km} / \mathrm{h}$ line is being planned. When train speed increases to $160 \mathrm{~km} / \mathrm{h}$, the eddy current becomes significant, resulting in reduced levitation force.

In this paper, a mathematical model of electromagnet is established, and the mechanism and influence factors of the eddy current are analyzed. The electromagnet model is analyzed by three-dimensional transient magnetic field of professional electromagnet simulation software Maxwell. The method of compensating the eddy current effect is put forward according to the decrease of the levitation force. 


\section{MECHANISM OF EDDY CURRENT}

According to Faraday's law of electromagnetic induction, the movement of a conductor in a changing magnetic field or a conductor in a magnetic field can cause an induced electromotive force or an induced current in the conductor, and this phenomenon is called the eddy effect. Non-laminated steel rails are normally used in the low speed maglev system and eddy current in the rail is easily induced by moving magnets[1]. The eddy current will weaken the air gap magnetic field and reduce the levitation force.

Japanese scholar Yamamura has performed a theoretical analysis on the eddy current effect of the train electromagnet. The analytical solution of the suspension force is given under the conditions that magnetic flux leakage, rail nonlinearity and orbital flux saturation are neglected. Its model and calculation formula are as follows [2]:
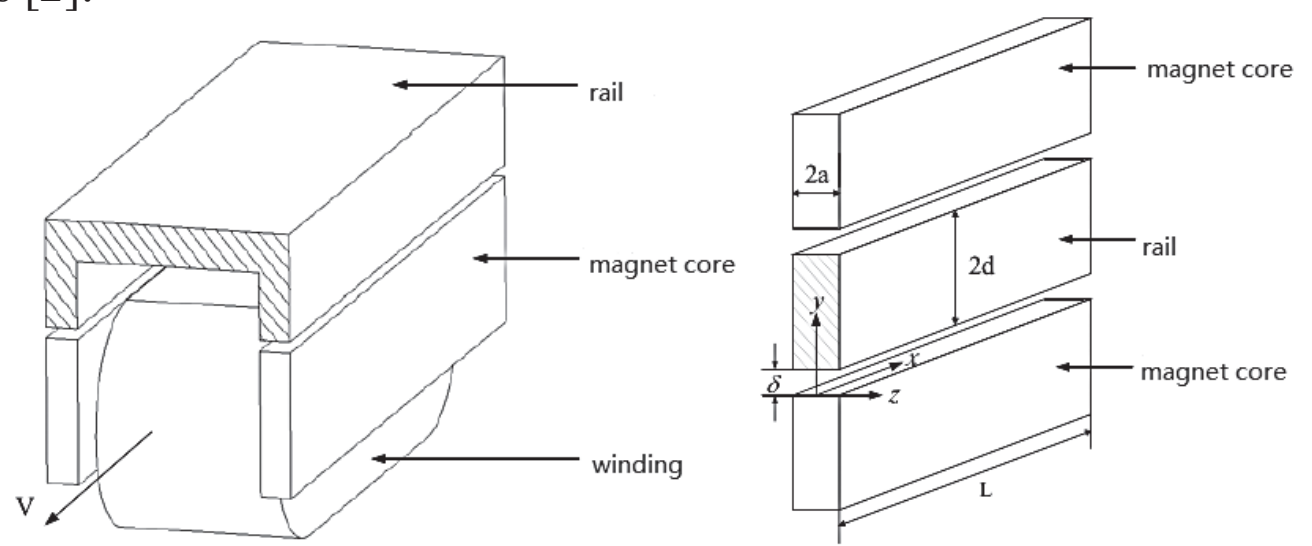

Fig. 1. Simplified model of electromagnet

$$
\begin{gathered}
F_{y o}=\frac{1}{\mu_{0}} \int_{0}^{L} d x \int_{-a}^{a} B_{0} d z=a L B_{0}{ }^{2} / \mu_{0} \\
F_{y}=F_{y o} \sum_{n=1}^{\infty} C_{n}^{2} \frac{1}{2 L\left[L-\frac{1}{a_{n}\left(1-e^{-a_{n} L}\right)}\right]} \\
C_{n}=\frac{4}{(2 n-1) \pi} \sin \frac{\pi(2 n-1)}{2} \\
a_{n}=\frac{\gamma_{n}=\frac{(2 n-1) \pi}{2 a}}{2\left(-K+\sqrt{K^{2}+4 \gamma_{n}^{2}}\right)}
\end{gathered}
$$




$$
K=\frac{\sigma \mu_{0} d v}{\delta}
$$

$F_{y}$ - levitation force, $F_{y o}$ - stationary levitation force, $\mu_{0}$ - magnetic permeability in vacuum, $B_{0}$ - magnetic flux density in air gap, $L$ - length of iron core, $2 \mathrm{a}$ - rail thickness, $d$-single-side rail height, $\delta$ - levitation gap, $\sigma$ - electrical conductivity.

From the calculation formula, it can be concluded that the levitation force is related to five parameters, such as speed, length of the electromagnet, rail height, rail thickness and air gap. Fig. 2 shows the variation of levitation force $F_{y} / F_{y o}$ with velocity $[3,4]$.

Fig. 2 (a) shows that the levitation force of electromagnet decreases with the increase of train speed.

Fig. 2 (b) shows the influence of electromagnet length on the eddy current effect to levitation force.

Fig. 2 (c) shows that larger the rail thickness, smaller the influence of the eddy current on the levitation force.

Fig. 2 (d) shows the influence of the eddy current on the levitation force with different values of air gap.

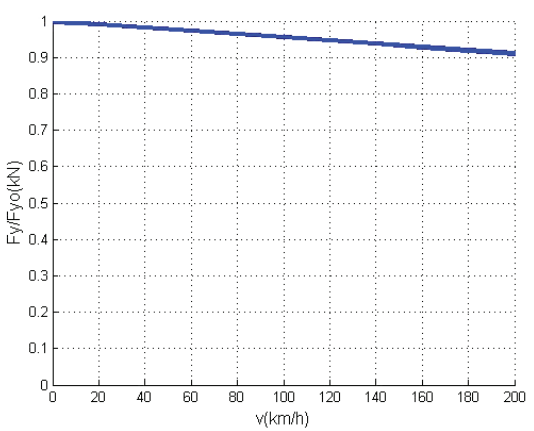

(a) levitation force - speed

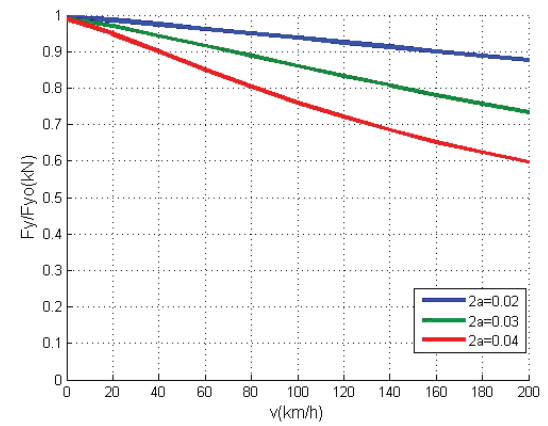

(c) levitation force - speed with different rail thickness

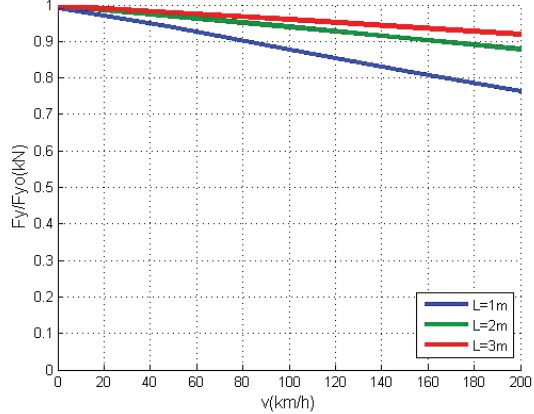

(b) levitation force - speed with different lengths of electromagnet

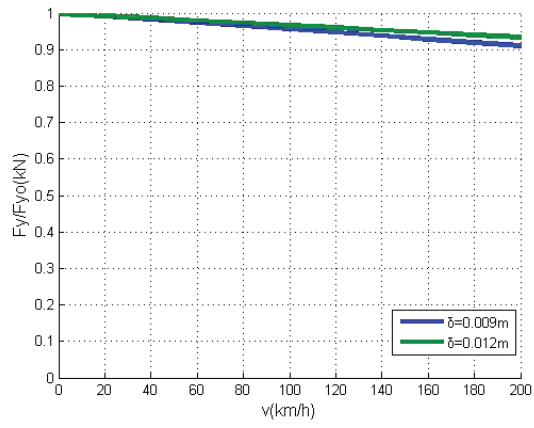

(d) levitation force - speed with different air gaps

Fig. 2. $F_{y} / F_{y o}$ decreases with increasing speed 


\section{FINITE ELEMENT SIMULATION}

Electromagnet parameters are show in table 1.

Table 1. Electromagnet parameters

\begin{tabular}{|l|c|}
\hline \multicolumn{1}{|c|}{ Parameters } & Values \\
\hline Rail thichness $(2 \mathrm{a})$ & $0.028 \mathrm{~m}$ \\
\hline Magnet Length $(L)$ & $2.72 \mathrm{~m}$ \\
\hline Air gap $(\delta)$ & $0.009 \mathrm{~m}$ \\
\hline Ampere-turns $(i)$ & $15360 \mathrm{~A}$ \\
\hline
\end{tabular}

The three-dimensional transient simulation model of electromagnet is established by Maxwell simulation software. As shown in Figure 3, the moving object is an electromagnet, the electromagnet is moving in the direction of the $\mathrm{X}$ axis. The $\mathrm{V}$ shows the direction of the electromagnet in Figure 3, and the simulation assumes that the electromagnet and the rail have no lateral misplacement and side rolling.

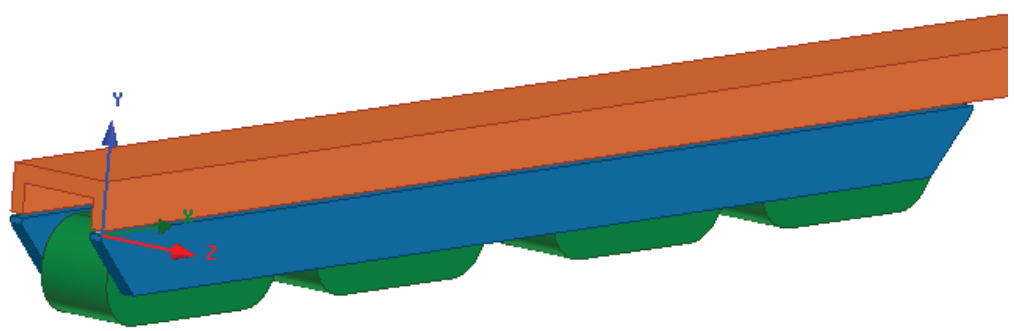

Fig. 3. Simulation model of electromagnet module

The $\mathrm{Jz}$ distribution of lateral density of rail eddy current density at different speeds is shown in Fig. 4. The eddy current density at the two ends of the electromagnet is larger than that of in the middle part of the electromagnet. The eddy current at both ends is in opposite direction and increases with the speed. The peak position at both ends does not vary with speed. The front end of the eddy current is in the negative direction along the $\mathrm{Y}$ axis and the back end is in the direction of the $\mathrm{Y}$ axis. This indicates that the eddy current weakens the front magnetic field of the electromagnet and strengthens the magnetic field at the end of the electromagnet [5].

The density of magnetic flux By distribution of the vertical component in the air gap at different speeds is shown in Fig. 5. The By of the electromagnet decreases with increasing eddy current in the front of the air gap, and the By changes very little at the end. Comparing the air gap flux density at different speed, it indicates that the average magnetic flux density is smaller in the air gap when the velocity 


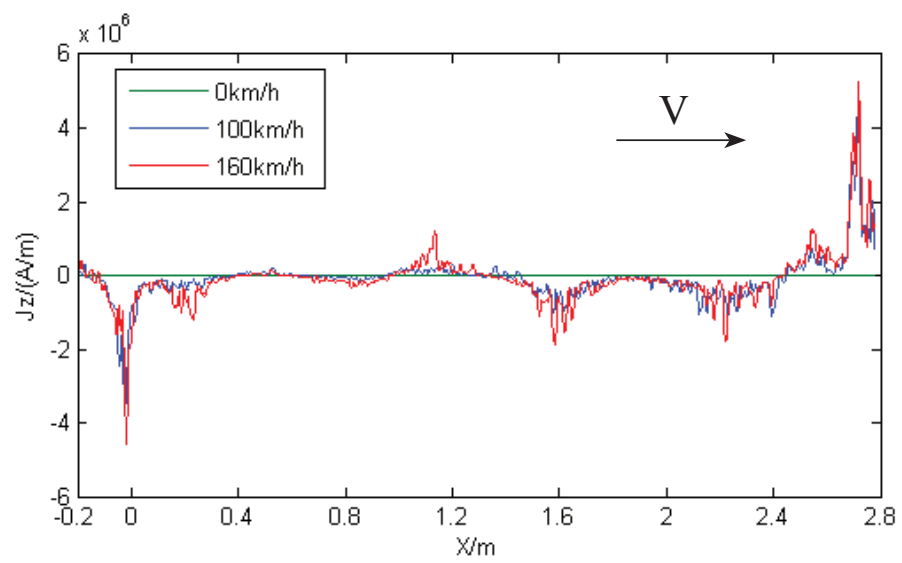

Fig. 4. Distribution of eddy density lateral component Jz at different speeds

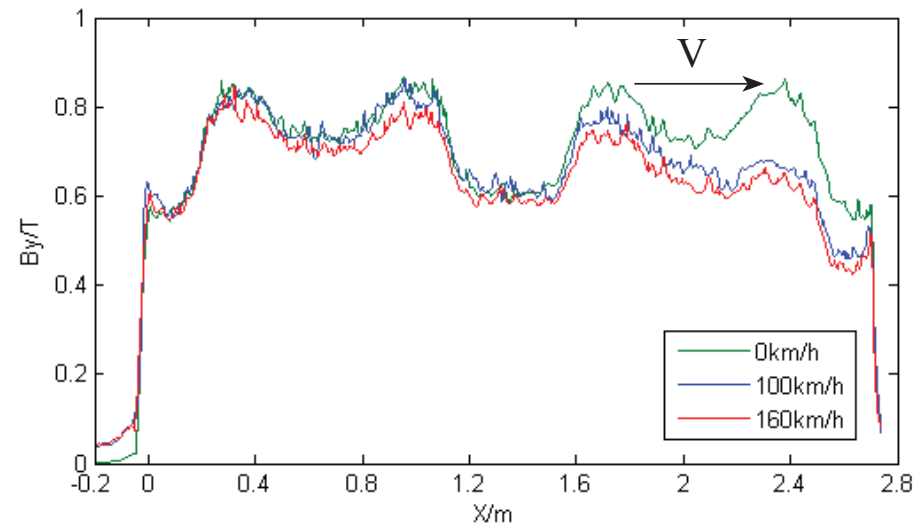

Fig. 5. Distribution of vertical component by of air gap flux density at different speeds

is larger. The decrease of the air gap means that flux density will directly reduce levitation force. The higher the speed of the electromagnet, the more significant the effect of eddy current, the more the lead force is reduced [5].

The levitation force of the electromagnet model is calculated, and the results of the finite element simulation is shown in Table 2 . When the speed is $160 \mathrm{~km} / \mathrm{h}$, the levitation force of the electromagnet decreases by $21.9 \%$ compared with $0 \mathrm{~km} / \mathrm{h}$. The current may increase by about $6 \mathrm{~A}$ to meet the requirements, with the same air gap and vehicle weight. The increasing current will lead to higher temperature which can damage the electromagnet. Therefore, reducing the effect of eddy current is the key to achieve higher speed.

Table 2. Simulation results

\begin{tabular}{|c|c|c|c|}
\hline $\begin{array}{c}\text { Speed, } \\
\mathbf{k m} / \mathbf{h}\end{array}$ & $\begin{array}{c}\text { Levitation } \\
\text { force, } \mathbf{k N}\end{array}$ & $\begin{array}{c}\text { Descent range, } \\
\mathbf{~ k N}\end{array}$ & $\begin{array}{c}\text { Descent rate comparing } \\
\text { to stationary, } \%\end{array}$ \\
\hline 0 & 39.6 & 0 & 0 \\
\hline 100 & 33.9 & 5.7 & 14.4 \\
\hline 160 & 30.9 & 8.7 & 21.9 \\
\hline
\end{tabular}




\section{OPTIMUM DESIGN OF ELECTROMAGNET}

In order to compensate the influence of eddy current effect on the levitation force, the electromagnet must be optimized. According to the analytical results mentioned above, influence of the eddy current on the levitation force can be reduced by three parameters: electromagnet length, the rail height and rail thickness. The rail height is determined by the overall scheme of the line, which is generally difficult to change. Although the influence of the eddy current on the levitation force can be reduced through decreasing the rail thickness, the levitation force will also be reduced. The purpose of this paper is to compensate the eddy current effect on the levitation force by extending the core length and adding extra coils.

Based on the original electromagnet scheme, length of iron core at the front end is increased and an electromagnet coil is also added, as shown in Fig. 6.

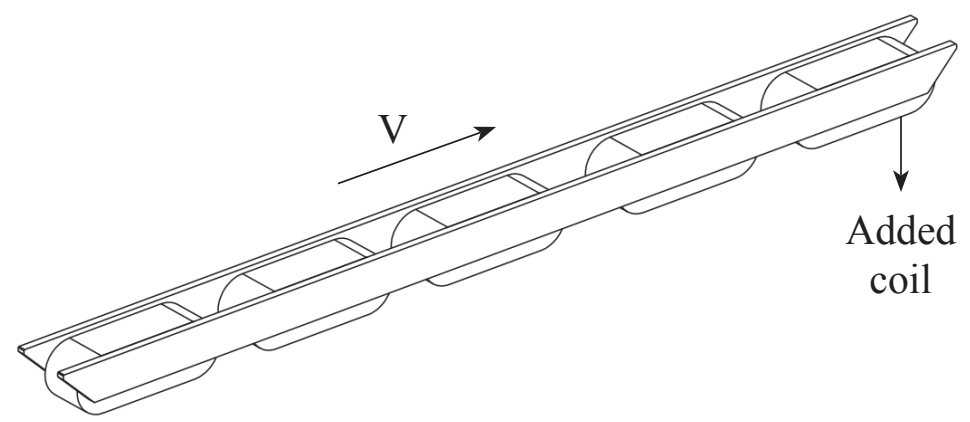

Fig. 6. The optimized electromagnet

The levitation force of the optimized electromagnet increases at speed of $0 \mathrm{~km} / \mathrm{h}$, which can still meet the requirement of vehicle weight after the eddy current is weakened. The benefits of the scheme are as follows: simple structure, easy maintenance, constant installation interface and flexible disassembly and assembly according to the actual demand of the train.

The optimization electromagnet simulation model is established, and the simulation results are shown in Table 3.

Table 3. Simulation results on levitation force of electromagnet

\begin{tabular}{|c|c|c|c|}
\hline $\begin{array}{c}\text { Speed, } \\
\mathbf{k m} / \mathbf{h}\end{array}$ & $\begin{array}{c}\text { levitation } \\
\text { force, } \mathbf{k N}\end{array}$ & $\begin{array}{c}\text { Descent range, } \\
\mathbf{~ k N}\end{array}$ & $\begin{array}{c}\text { Descent rate comparing } \\
\text { to stationary, } \%\end{array}$ \\
\hline 0 & 50.4 & 0 & 0 \\
\hline 50 & 48.2 & 2.2 & 4.4 \\
\hline 100 & 45.1 & 5.3 & 10.5 \\
\hline 160 & 41.4 & 9.0 & 17.9 \\
\hline 200 & 39.3 & 11.1 & 22.1 \\
\hline
\end{tabular}


The levitation force is $41.4 \mathrm{kN}$ at the speed of $160 \mathrm{~km} / \mathrm{h}$, which is slightly larger than the $39.6 \mathrm{kN}$ of the former electromagnet static levitation force, which can meet the requirements.

\section{CONCLUSION}

Reducing the effect of eddy current on levitation force is the key to further speed up the low speed suspension train. Starting from the analytical method, the generation mechanism and influencing factors of rail eddy current effect are analyzed. The three-dimensional transient magnetic field simulation of the electromagnet model is carried out by the Maxwell software, and the decrease of levitation force at different speeds is obtained. According to the simulation results, the electromagnet is optimized. A method of extending the iron core length and adding extra coils in the front of electromagnet is proposed to compensate the loss of the levitation force.

\section{References}

1. Du J, Ohsaki H. Numerical Analysis of Eddy Current in the EMS-Maglev System. Proceeding of The $1^{\text {st }}$ International Conference on Electrical Machines and Systems (ICEMS2003), November 2003.

2. Yamamura S, Ito T. Analysis of Speed Characteristics of Attracting Magnet for Magnetic Levitated Vechicles. IEEE Trans on Magnetics. 1975;11(5):1504-1507. doi: 10.1109/ tmag.1975.1058850

3. Ohsaki H, Du J. Influence of eddy current induced in the steel rails on electromagnetic force characteristics of EMS maglev systems. Proceeding of the International Conference MAGLEV 2004, Oct 26-28, 2004, Shanghai. 2004;2:960-965.

4. Zheng L. Research on the Influence of Eddy Current Induced in Steel Rails on the Levitation Force of a Maglev Train. Changsha: National University of Defense Technology, 2010.

5. Zheng Lili, Li Jie, Li Jinhu. Research on Influence of Eddy Current Induced in Steel Rails on Levitation Force of Maglev. Computer Simulation. 2011;28(8):328-331.

Information about the authors:

Liang Fang, Master, Engineer;

ORCID: 0000-0001-8874-9208;

E-mail: fangliang@crrcelectric.com

Yunfeng He, Ph.D, senior engineer;

ORCID: 0000-0002-9951-3919;

E-mail: heyunfeng@crrcelectric.com

Lei Zhang, Master, Engineer;

ORCID: 0000-0003-0840-9401;

E-mail: zhanglei@crrcelectric.com

To cite this article:

Fang L, He Y, Zhang L. Research on Mechanism of Eddy Current in Rail of Maglev and Optimum Design of Electromagnet. Transportation Systems and Technology. 2018;4(3 suppl. 1):272-278. doi: $10.17816 /$ transsyst201843s1272-278 